\title{
How long does treatment with fixed orthodontic appliances last? A systematic review
}

\author{
Aliki Tsichlaki, ${ }^{a}$ Siew Yee Chin, ${ }^{b}$ Nikolaos Pandis, ${ }^{c}$ and Padhraig S. Fleming ${ }^{d}$ \\ London, United Kingdom, Corfu, Greece and Bern, Switzerland
}

\begin{abstract}
Introduction: There is little agreement on the expected duration of a course of orthodontic treatment; however, a consensus appears to have emerged that fixed appliance treatment is overly lengthy. This has spawned numerous novel approaches directed at reducing the duration of treatment, occasionally with an acceptance that occlusal outcomes may be compromised. The aim of this study was to determine the mean duration and the number of visits required for comprehensive orthodontic treatment involving fixed appliances. Methods: Multiple electronic databases were searched with no language restrictions, authors were contacted as required, and reference lists of potentially relevant studies were screened. Randomized controlled trials and nonrandomized prospective studies concerning fixed appliance treatment with treatment duration as an outcome measure were included. Data extraction and quality assessment were performed independently and in duplicate. Results: Twenty-five studies were included after screening: 20 randomized controlled trials and 5 controlled clinical trials. Twenty-two studies were eligible for meta-analysis after quality assessment. The mean treatment duration derived from the 22 included studies involving 1089 participants was 19.9 months (95\% confidence interval, $19.58,20.22$ months). Sensitivity analyses were carried out including 3 additional studies, resulting in average duration of treatment of 20.02 months (95\% confidence interval, 19.71, 20.32 months) based on data from 1211 participants. The mean number of required visits derived from 5 studies was 17.81 (95\% confidence interval, 15.47, 20.15 visits). Conclusions: Based on prospective studies carried out in university settings, comprehensive orthodontic treatment on average requires less than 2 years to complete. (Am $\mathrm{J}$ Orthod Dentofacial Orthop 2016;
\end{abstract}

$\mathrm{I}$ $\mathrm{t}$ is accepted that comprehensive orthodontic treatment is lengthy; the time frame is largely dictated by the biologic principles underpinning optimal tooth movement. ${ }^{1,2}$ There has been a lack of clarity concerning the typical duration of treatment. In a previous review that included observational studies, the authors were unable to arrive at an overall estimate of treatment duration. ${ }^{3}$ In spite of this lack of

\footnotetext{
${ }^{a}$ Senior registrar, Barts and the London School of Medicine and Dentistry, Queen Mary University, London, United Kingdom.

${ }^{\mathrm{b}}$ Registrar, Barts and the London School of Medicine and Dentistry, Queen Mary University, London, United Kingdom.

'Private practice, Corfu, Greece; visiting assistant professor, Department of Orthodontics, Dental School, Medical Faculty, University of Bern, Bern, Switzerland.

${ }^{\mathrm{d}}$ Senior clinical lecturer and consultant, Barts and the London School of Medicine and Dentistry, Queen Mary University, London, United Kingdom.

All authors have completed and submitted the ICMJE Form for Disclosure of Potential Conflicts of Interest, and none were reported.

Address correspondence to: Padhraig S. Fleming, Department of Orthodontics, Barts and the London School of Medicine and Dentistry, Institute of Dentistry, Queen Mary University of London, Turner St, London E1 2AD United Kingdom; e-mail, padhraig.fleming@gmail.com.

Submitted, revised and accepted, September 2015.

0889-5406/ $\$ 36.00$

Copyright (C) 2016 by the American Association of Orthodontists.

http://dx.doi.org/10.1016/j.ajodo.2015.09.020
}

a clear yardstick, there has been a seemingly relentless drive among orthodontists and general dentists to reduce the duration of orthodontic treatment. Modern adjuncts directed at hastening treatment include newer technologies and novel surgical procedures, but some clinicians also resort to eschewing integral treatment phases in an effort to reduce treatment times. ${ }^{4,5}$

Excessive treatment duration has been linked to a greater susceptibility to iatrogenic consequences of appliance therapy, primarily root resorption and plaque-induced conditions, including demineralization. ${ }^{6}$ Moreover, patient compliance and oral healthrelated quality of life may be impaired by longer treatment, particularly in adults. ${ }^{7}$ Shorter treatment times may, therefore, theoretically offer advantages to both treatment providers and patients, although shorter treatment is not without significant potential disadvantages.

For providers of care, there may be financial incentives in delivering more efficient treatment, most likely associated with fewer visits and shorter chairside times. ${ }^{8}$ However, potential financial gain may be tempered by the necessity for prolonged and diligent retention associated with the placement of teeth in inherently unstable 
positions with limited torque expression when the objectives of treatment are confined to the alignment of anterior teeth in isolation. ${ }^{5}$

Novel approaches, involving various degrees of financial outlay and theoretical risk, have included expensive vibratory appliances ${ }^{9}$ and adjunctive surgical procedures to expedite tooth movement. ${ }^{10}$ Both, however, appear to be largely unproven; a randomized trial failed to identify an increase in the rate of orthodontic alignment in conjunction with a well-marketed, nonsurgical adjunct involving vibratory stimulation. ${ }^{11}$

Moreover, a recent Cochrane review highlighted a lack of evidence to support the use of surgical adjuncts at this stage, with only 4 clinical trials incorporating a total of just 57 patients. ${ }^{12}$ Furthermore, patient perceptions of surgically assisted orthodontics are not all favorable, especially when given the alternative of other noninvasive techniques. ${ }^{13}$

It is therefore increasingly important that there is an appreciation of the expected length of orthodontic treatment before routinely embarking on treatment involving compromised objectives or adjunctive procedures, particularly with the lack of evidence underpinning these approaches. The aim of our review was to determine the duration of orthodontic treatment with fixed appliances.

\section{MATERIAL AND METHODS}

The protocol for this systematic review was registered on PROSPERO international prospective register of systematic reviews (www.crd.york.ac.uk/prospero; protocol, I CRD42014014983). The following inclusion and exclusion criteria were used.

1. Study design. Randomized and prospective nonrandomized studies carried out in primary or secondary care or in the community were to be included. Studies with short follow-up periods not including the duration of orthodontic treatment and retrospective studies were excluded.

2. Participants. Patients of any age with completearch, fixed, bonded orthodontic appliances followed until the end of treatment were to be included. Patients with craniofacial syndromes and cleft lip or palate were excluded.

3. Interventions and comparators. Any treatment intervention involving comprehensive, completearch, fixed orthodontic appliances without adjunctive use of removable or functional appliances was included. Patients undergoing treatment involving fixed appliances with surgical interventions including surgical exposure of ectopic teeth were excluded. Interceptive orthodontic interventions were also excluded. Since this was an epidemiologic review, no between-group comparisons were planned.

4. Outcome measures. These were the duration of orthodontic treatment (months) from appliance placement to removal and the number of visits.

\section{Search strategy for identification of studies}

Comprehensive electronic database searches were undertaken without language restrictions as follows: MEDLINE via OVID (to November 2014, Appendix), the Cochrane Oral Health Group's Trials Register (November 2014), and the Cochrane Central Register of Controlled Trials (CENTRAL, the Cochrane Library lssue 3, 2014). Unpublished literature was accessed electronically through ClinicalTrials.gov (www.clinicaltrials.gov) and the National Research Register (www.controlled-trials. com) using the term orthodontic. In addition, efforts were made to obtain conference proceedings and abstracts, with authors contacted to identify unpublished or ongoing clinical trials. Reference lists of included studies were screened for additional relevant research.

\section{Assessment of relevance, validity, and data extraction}

Data were extracted independently and in duplicate by 2 authors (A.T., S.Y.C.) using prepiloted data extraction forms. The investigators were not blinded to the authors or the results of the research, and any disagreements were resolved by discussion with a third author (P.S.F.). The following information was recorded where available: (1) year of publication and study setting; (2) participants: sample size, age, and sex; (3) type of intervention; (4) type of control; and (5) outcomes: treatment duration (including means and standard deviations in months, where available) and number of visits (means).

Authors were contacted to clarify data as required, including information on treatment duration.

The quality of the eligible trials was assessed independently and in duplicate by 2 authors (A.T., S.Y.C.), and any disagreements were resolved by discussion with a third reviewer (P.S.F.). The Cochrane Collaboration's risk of bias tool was used to assess risk of bias for randomized controlled trials (RCTs), ${ }^{14}$ and the Newcastle-Ottawa scale was used for the nonrandomized studies. ${ }^{15}$ The following domains were assessed as being at low, high, or unclear risk of bias for the RCTs: sequence generation (selection bias), allocation concealment (selection bias), blinding of participants and personnel (performance bias) and outcome assessors (detection bias), incomplete outcome data addressed 


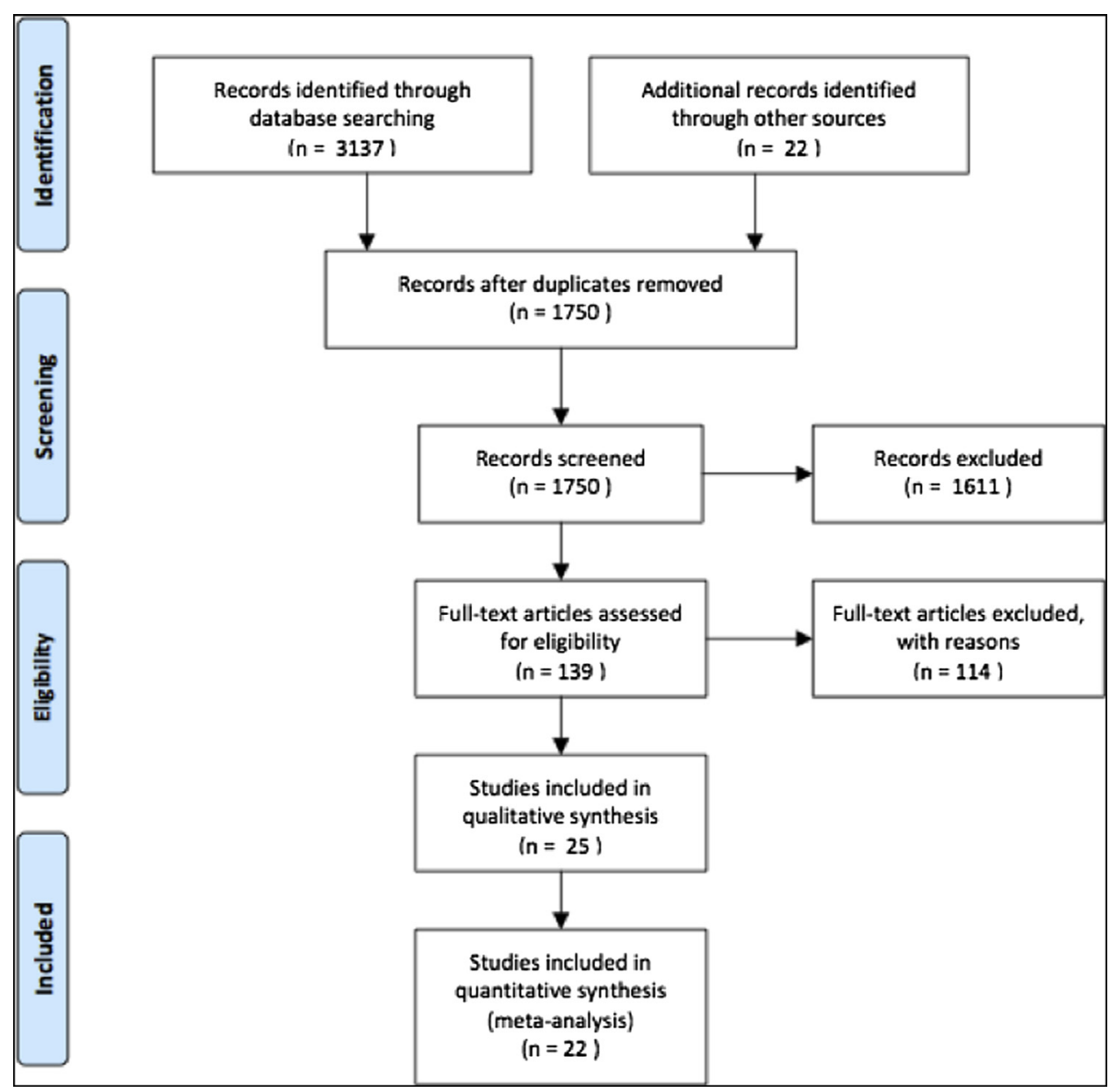

Fig 1. PRISMA flowchart of article identification and selection.

(attrition bias), selective outcome reporting (reporting bias), and other biases. An overall assessment of risk of bias (high, unclear, low) was made for each included trial. Studies with at least 1 criterion considered to be at high risk of bias were considered to be at high risk of bias overall and excluded from the primary analysis. The nonrandomized studies were judged on 3 broad perspectives consisting of 8 domains: selection of the study groups, comparability of the groups, and ascertainment of the outcome of interest. A star system was used in which high-quality studies could receive a maximum of 9 stars. A rating of 5 stars or fewer signified low quality. Studies at high risk of bias and low quality were excluded from the primary meta-analysis.

\section{Statistical analysis}

Clinical heterogeneity of the included studies was analyzed by reviewing the treatment interventions and protocol, including participants and settings, appliance designs, and use of extractions or additional procedures.
Statistical heterogeneity was assessed based on a graphic display of the estimated treatment durations in conjunction with 95\% confidence intervals. A weighted estimate of treatment duration was calculated from the included studies. Sensitivity analyses were planned at the outset to address studies at higher risk of bias and other potential sources of heterogeneity including overriding effects of large studies and differences in outcomes related to specific treatments (eg, extraction vs nonextraction), study setting (primary or secondary care), or patient groups (adults over 18 years vs adolescents). Metaanalyses and sensitivity analyses were undertaken with Stata software (StataCorp, College Station, Tex) using metan and metareg commands.

\section{RESULTS}

The search returned 1728 studies after removal of duplicates. All abstracts were reviewed, and 139 potentially relevant articles were retrieved in full. Additional screening of reference lists returned another 22 articles 


\begin{tabular}{|c|c|c|c|c|c|}
\hline Study & Design & Participants & Intervention/comparison & $\begin{array}{l}\text { Extraction } \\
\text { (yes/no) }\end{array}$ & $\begin{array}{l}\text { Adults/young } \\
\text { people }(<18 \text { y) }\end{array}$ \\
\hline Al Maaitah, $2013^{16}$ & $\begin{array}{l}\text { RCT } \\
\text { split mouth }\end{array}$ & $\begin{array}{l}\mathrm{N}=34: 204 \text { brackets SEP and } 204 \text { brackets conventional etch. Each } \\
\text { group split in } \leq 17 \mathrm{y} \text { and }>17 \mathrm{y} \text {. Overall mean age, } 18.7 \mathrm{y} \text {; range, } \\
12-26 \mathrm{y} ; \text { sex, } 13 \mathrm{M}, 21 \mathrm{~F}\end{array}$ & SEP vs conventional etch & No & Both \\
\hline Banks, ${ }^{17} 2000$ & CCT & $\begin{array}{l}\mathrm{N}=94: 49 \text { fluoride- releasing modules (782 teeth) and } 45 \\
\text { nonfluoride-releasing modules ( } 740 \text { teeth). Overall mean age, } 16 \text { y }\end{array}$ & $\begin{array}{l}\text { Fluoride-releasing modules vs } \\
\text { nonfluoride- releasing modules }\end{array}$ & No & Both \\
\hline Borsos, ${ }^{18} 2012$ & CCT & $\begin{array}{l}\mathrm{N}=30: 15 \text { palatal implant }(\mathrm{Pl}) \text { and } 15 \text { transpalatal arch (TPA). Overall } \\
\text { mean age, } 14.22 \pm 1.37 \mathrm{y}\end{array}$ & Pl vs TPA & Yes & Young \\
\hline Cattaneo, ${ }^{19} 2011$ & RCT & $\begin{array}{l}\mathrm{N}=41: 20 \text { active ligation } \ln \text {-Ovation } \mathrm{R} \text { and } 21 \text { passive ligation Damon } \\
\quad 3 \mathrm{MX}\end{array}$ & $\begin{array}{l}\text { In-0vation R (active) vs Damon } \\
3 \mathrm{MX} \text { (passive) self-ligation }\end{array}$ & No & Both \\
\hline DiBiase, ${ }^{20} 2011$ & RCT & $\begin{array}{l}\mathrm{N}=48: 27 \text { Damon } 3 \text { and } 21 \text { Synthesis. Overall mean age, } 16.2 \mathrm{y} \text {; sex, } \\
32 \mathrm{M}, 30 \mathrm{~F} \text { initially }\end{array}$ & $\begin{array}{l}\text { Damon } 3 \text { self-ligation vs Synthesis } \\
\text { conventional ligation }\end{array}$ & Yes & Both \\
\hline Fleming, ${ }^{21} 2010$ & RCT & $\begin{array}{l}\mathrm{N}=54: 28 \text { SmartClip self-ligation and } 26 \text { Victory conventional ligation. } \\
\text { Mean overall age, } 15.81 \pm 2.58 \mathrm{y} ; \text { sex, } 18 \mathrm{M}, 36 \mathrm{~F}\end{array}$ & $\begin{array}{l}\text { SmartClip self-ligation vs Victory } \\
\text { conventional ligation }\end{array}$ & Yes & Both \\
\hline Germec, $^{22} 2008$ & RCT & $\mathrm{N}=26: 13$ extraction and 13 nonextraction & Extraction vs air rotor stripping & Yes & Both \\
\hline Jenatschke, ${ }^{23} 2001$ & $\begin{array}{l}\text { RCT } \\
\text { (feasibly study) }\end{array}$ & $\begin{array}{l}\mathrm{N}=33: 18 \text { chlorhexidine varnish }(\mathrm{CHX}) \text { and } 15 \text { placebo varnish. Median } \\
\text { overall age, } 15 \mathrm{y} \text {; range, } 11-18 \mathrm{y}\end{array}$ & $\begin{array}{l}\text { CHX varnish vs placebo varnish } \\
\text { group }\end{array}$ & Unclear & Both \\
\hline Jiang, ${ }^{24} 2013$ & $\mathrm{RCT}$ & $\mathrm{N}=95: 48$ acidulated phosphate fluoride (APF) and 47 placebo & $1.23 \%$ APF vs placebo & Unclear & Young \\
\hline Johansson, ${ }^{25} 2012$ & RCT & $\begin{array}{l}\mathrm{N}=90: 44 \text { Time } 2 \text { self ligation (SL) and } 46 \text { Gemini conventional } \\
\text { edgewise (CE). Overall age range, } 11.7-18.2 \text { y }\end{array}$ & Time2 SL vs Gemini CE & No & Both \\
\hline Jung, ${ }^{26} 2013$ & CCT & $\begin{array}{l}\mathrm{N}=66: 34 \text { orthodontic mini-implant (OMI) with intreproximal stripping } \\
\text { (IPS) and } 32 \text { second premolar extraction. Overall age range, 17-44 y }\end{array}$ & $\mathrm{OMl}+\mathrm{IPR}$ vs extraction & Yes & Adults \\
\hline Liu, ${ }^{27} 2009$ & RCT & $\mathrm{N}=34: 17$ mini-implant and 17 TPA. Sex, $6 \mathrm{M}, 28 \mathrm{~F}$ & Mini-implant vs TPA & Yes & Both \\
\hline $\mathrm{Ma},{ }^{28} 2008$ & RCT & $\begin{array}{l}\mathrm{N}=30: 15 \text { mini-implant and } 15 \text { headgear }(\mathrm{HG}) . \text { Overall age range, } \\
18-22 \mathrm{y} \text {; sex, } 16 \mathrm{M}, 14 \mathrm{~F}\end{array}$ & Mini-implant vs HG & Yes & Adults \\
\hline Magnius, ${ }^{29} 2014$ & $\begin{array}{l}\text { RCT } \\
\text { split mouth }\end{array}$ & $\begin{array}{l}\mathrm{N}=46 \text { (836 teeth): } 416 \text { teeth pumice and } 416 \text { teeth prophy paste. } \\
\text { Overall mean age, } 14.1 \pm 1.4 \mathrm{y} \text {; sex, } 17 \mathrm{M}, 29 \mathrm{~F}\end{array}$ & Oil-free pumice vs prophy paste & Yes & Young \\
\hline Manning, ${ }^{30} 2006$ & $\mathrm{RCT}$ & $\begin{array}{l}\mathrm{N}=34: 17 \text { ( } 299 \text { bonds) SEP and } 17 \text { ( } 298 \text { bonds) Transbond conventional } \\
\text { adhesive }\end{array}$ & $\begin{array}{l}\text { SEP vs conventional adhesive } \\
\text { (Transbond) }\end{array}$ & Yes & Young \\
\hline Miller, $^{31} 1996$ & RCT & $\mathrm{N}=17: 9 \mathrm{GlC}$ and 8 composite bracket adhesive & GIC vs resin adhesive & Unclear & Young \\
\hline Millett, ${ }^{32} 1999$ & $\begin{array}{l}\text { CCT } \\
\text { split mouth }\end{array}$ & $\begin{array}{l}\mathrm{N}=40 \text { ( } 240 \text { brackets): } 120 \text { brackets } \mathrm{GlC} \text { and } 120 \text { resin. Overall mean age, } \\
13.4 \pm 2 \mathrm{y} ; \text { sex, } 17 \mathrm{M}, 23 \mathrm{~F}\end{array}$ & GlC vs resin adhesive & Unclear & Young \\
\hline Millett, ${ }^{33} 2000$ & $\begin{array}{l}\text { CCT } \\
\text { split mouth }\end{array}$ & $\begin{array}{l}\mathrm{N}=45 \text { ( } 426 \text { brackets): } 213 \text { compomer and } 213 \text { resin adhesive. Overall } \\
\text { median age, } 14.4 \mathrm{y} \text {, range, 13.7-15.5 y; sex, } 13 \mathrm{M}, 32 \mathrm{~F}\end{array}$ & Compomer vs resin adhesive & No & Young \\
\hline Norevall, $^{34} 1996$ & $\begin{array}{l}\text { RCT } \\
\text { split mouth }\end{array}$ & $\begin{array}{l}\mathrm{N}=60: 30 \text { machine cut grooves bracket base and } 30 \text { mesh foil bracket } \\
\text { base; } 492 \text { teeth Aquacem }(\mathrm{GlC}) \text { and } 493 \text { Unite (resin). Overall mean } \\
\text { age, } 13.56 \pm 1.57 \mathrm{y} \text {; sex, } 21 \mathrm{M}, 39 \mathrm{~F}\end{array}$ & GIC vs acrylic resin & Yes & Young \\
\hline Polat, $^{35} 2008$ & $\mathrm{RCT}$ & $\begin{array}{l}\mathrm{N}=20: 10 \mathrm{ln}-\mathrm{Ovation} \mathrm{SL} \text { and } 10 \text { conventional preadusted edgewise } \\
\text { (CPAE) }\end{array}$ & SL vs CPAE & No & Young \\
\hline Reukers, $^{36} 1998$ & RCT & $\begin{array}{l}\mathrm{N}=61: 32 \text { fully programmed appliance (FPA) and } 29 \text { partly programmed } \\
\text { appliance (PPA). Overall mean age, } 12.4 \pm 1.2 \mathrm{y}\end{array}$ & $\begin{array}{l}\text { FPA SWA (0.022-in slot) vs PPA } \\
\text { (0.018-in slot) conventional } \\
\text { edgewise }\end{array}$ & Yes & Young \\
\hline
\end{tabular}




\section{Table. Continued}

\begin{tabular}{|c|c|c|c|c|c|}
\hline Study & Design & Participants & Intervention/comparison & $\begin{array}{l}\text { Extraction } \\
\text { (yes/no) }\end{array}$ & $\begin{array}{l}\text { Adults/young } \\
\text { people }(<18 \text { y) }\end{array}$ \\
\hline Sandler, ${ }^{37} 2008$ & RCT & $\begin{array}{l}N=51: 26 \text { palatal implant }(\mathrm{Pl}) \text { and } 25 \text { headgear }(\mathrm{HG}) \text {. Overall mean age, } \\
15.2 \mathrm{y} ; \text { range, 12-39 y; sex, } 13 \mathrm{M}, 38 \mathrm{~F}\end{array}$ & $\mathrm{Pl}$ vs HG & Yes & Young \\
\hline Sandler, ${ }^{38} 2014$ & RCT & $\begin{array}{l}\mathrm{N}=710.22 \text { TADs, } 26 \text { Nance, and } 23 \mathrm{HG} . \text { Overall mean age, } \\
14.22 \pm 1.46 \mathrm{y}\end{array}$ & TADs vs Nance palatal arch vs HG & Yes & Young \\
\hline Van der Veen, ${ }^{39} 2010$ & $\begin{array}{l}\text { RCT } \\
\text { split mouth }\end{array}$ & $\begin{array}{l}\mathrm{N}=28: 14 \text { buccal appliances maxilla with lingual mandible and } 14 \\
\text { lingual appliances maxilla with buccal appliances mandible. Overall } \\
\text { mean age, } 15.3 \pm 1.2 \mathrm{y}\end{array}$ & Buccal vs lingual appliances & Unclear & Both \\
\hline $\mathrm{Xu},{ }^{40} 2010$ & RCT & $\mathrm{N}=63: 32$ en-masse retraction and 31 2-step retraction. Sex, $24 \mathrm{M}, 39 \mathrm{~F}$ & $\begin{array}{l}\text { En-masse retraction vs } 2 \text {-step } \\
\text { retraction }\end{array}$ & Unclear & Young \\
\hline
\end{tabular}

SEP, Self-etching primer; $M$, male; $F$, female; $S W A$, straightwire appliance; TADs, temporary anchorage devices.

\begin{tabular}{|c|c|c|c|c|c|c|c|c|c|c|c|c|c|c|c|c|c|c|c|c|c|}
\hline 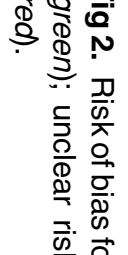 & $\begin{array}{l}\underset{\Xi}{\Xi} \\
\text { 号 } \\
\overrightarrow{0}\end{array}$ & 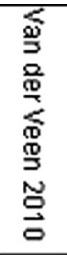 & 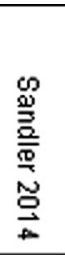 & 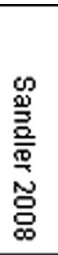 & 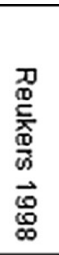 & 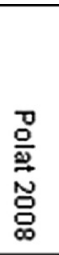 & 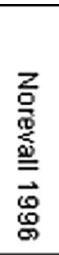 & 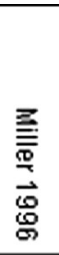 & 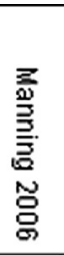 & 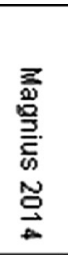 & 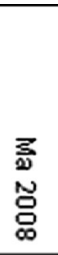 & 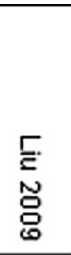 & 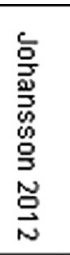 & 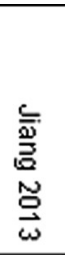 & 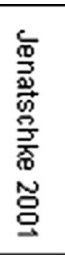 & 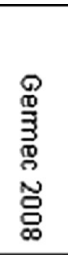 & 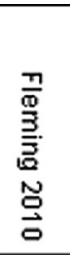 & 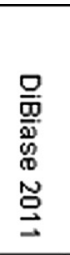 & 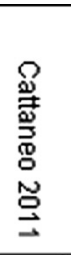 & 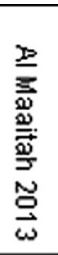 & \\
\hline 오 용 & + & + & + & + & + & $-v$ & $\cdot v$ & $\cdot v$ & + & + & + & + & + & + & $\sim$ & (1) & + & + & + & + & Random sequence generation (selection bias) \\
\hline 总. 光 & $\cdot v$ & $\cdot v$ & + & + & + & 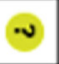 & $\cdot v$ & $\sim$ & $\cdot$ & $\sim$ & $\sim$ & $\sim$ & $\cdot$ & + & $\bullet$ & P & 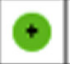 & $\sim$ & $\cdot$ & 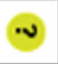 & Allocation concealment (selection bias) \\
\hline 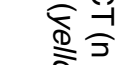 & + & + & + & + & + & + & $\cdot$ & + & + & $\sim$ & + & + & + & + & $\cdot$ & $\sim$ & 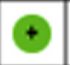 & + & $\sim$ & + & Blinding of participants and personnel (performance bias) \\
\hline 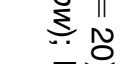 & $\hookrightarrow$ & $\cdot v$ & + & $\sim$ & $\omega$ & 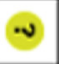 & $\cdot v$ & $\sim$ & $\cdot$ & + & $\omega$ & $\sim$ & + & + & $\bullet$ & $\sim$ & $-v$ & $\sim$ & + & $\sim$ & Blinding of outcome assessment (detection bias) \\
\hline 㕩 & + & + & + & + & + & $-v$ & $\cdot v$ & + & + & $\cdot$ & $\sim$ & $-\nu$ & + & + & + & + & 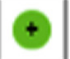 & + & + & $\cdot \nu$ & Incomplete outcome data (attrition bias) \\
\hline 元 & + & + & + & + & + & + & + & + & + & + & $\omega$ & + & + & + & + & + & + & + & + & + & Selectwe reporting (reporting bias) \\
\hline 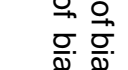 & + & + & + & + & $\oplus$ & + & $\cdot \nu$ & + & + & + & + & + & + & + & $\oplus$ & + & $\oplus$ & + & + & + & Other bias \\
\hline
\end{tabular}




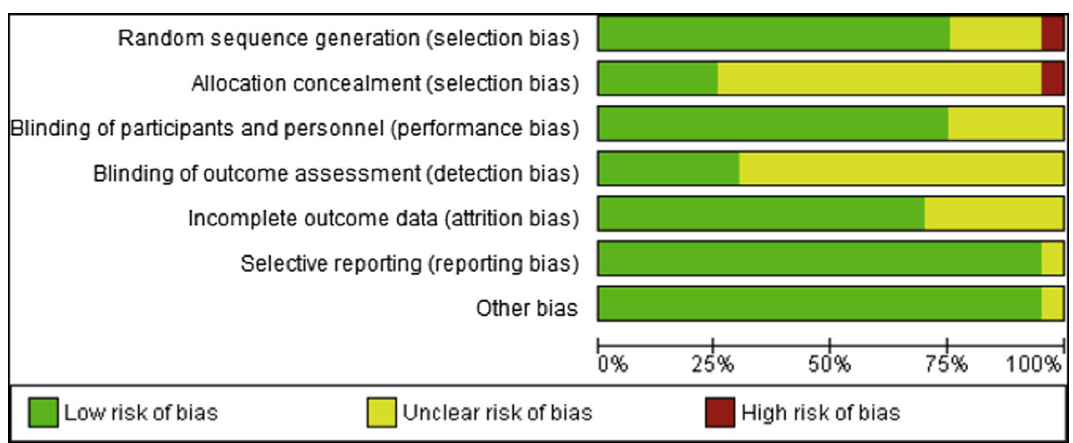

Fig 3. Overall risk of bias score for the specific domains.

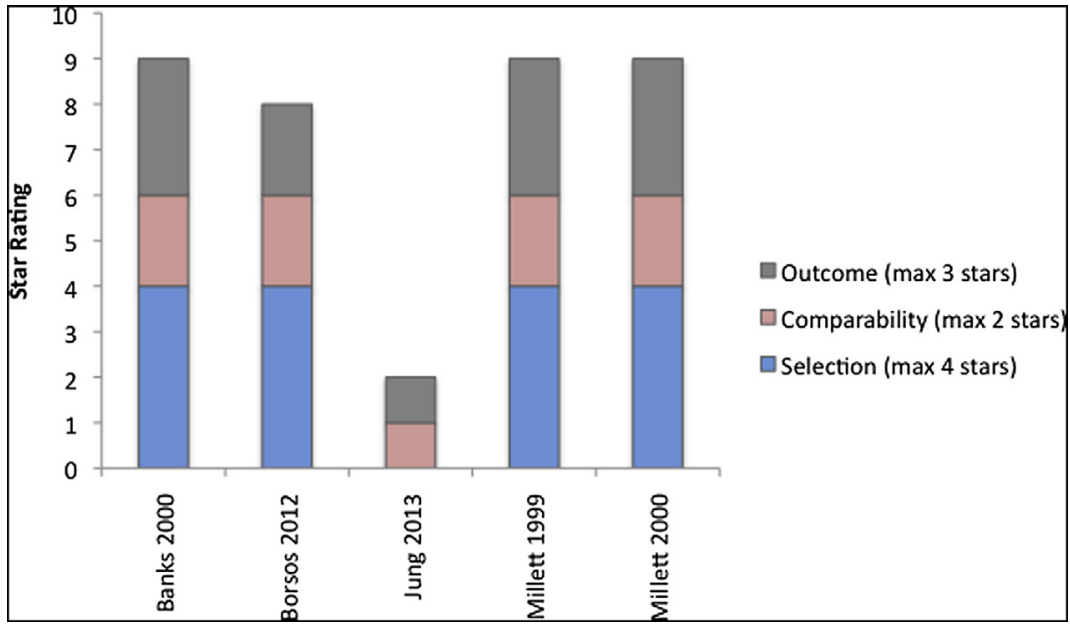

Fig 4. Newcastle-Ottawa scores for included nonrandomized studies $(n=5)$.

for review. After a detailed assessment, which included contacting the relevant authors for further clarification of data relating to treatment duration, 114 studies [F1-4/C] were excluded (Appendix), and 25 remained (Fig 1; Table ${ }^{16-40}$ ). Twenty studies were RCTs, and 5 were controlled clinical trials (CCTs). All included studies were carried out in a university or hospital setting. Among the primary studies, other primary outcomes of interest other than estimates of overall treatment duration, included bracket failure rates, prevalence of decalcification, and occlusal outcomes.

The generation of a random number sequence was considered adequate in 15 trials, with only 1 study [F3-4/C] considered at high risk of bias (Figs 2 and 3). Five studies were judged to have adequate allocation concealment, with allocation concealment not clearly reported in most studies. Blinding of the clinicians and patients to the intervention was not possible in many studies because of the nature of the research. Blinding of outcome assessors was possible; however, this was clearly undertaken in only 6 studies and was unclear in the remaining 14 RCTs. Overall, most of the included RCTs were deemed to be at low or unclear risk of bias. With regard to the CCTs, 4 studies were judged to be of good quality using the NewcastleOttawa scale (8-9 stars). One CCT was deemed to be of low quality (Fig 4).

The mean treatment duration derived from the 22 included studies involving 1089 participants was 19.9 months (95\% confidence interval [Cl], 19.58, 20.22 months) (Fig 5). Sensitivity analyses were carried [F5-4/C] out, including 3 additional studies; 1 study reported the duration of treatment with medians and ranges, and 2 studies were deemed to be of low quality overall. The resulting average duration of treatment based on the data from 1211 participants was 20.02 months (95\% Cl, 19.71, 20.3 months; Fig 6), indicating a similar [F6-4/C] result. In terms of visits required, this was reported in only 5 RCTs. The mean number of required visits was 17.81 (95\% Cl, 15.47, 20.15 visits; Appendix). 


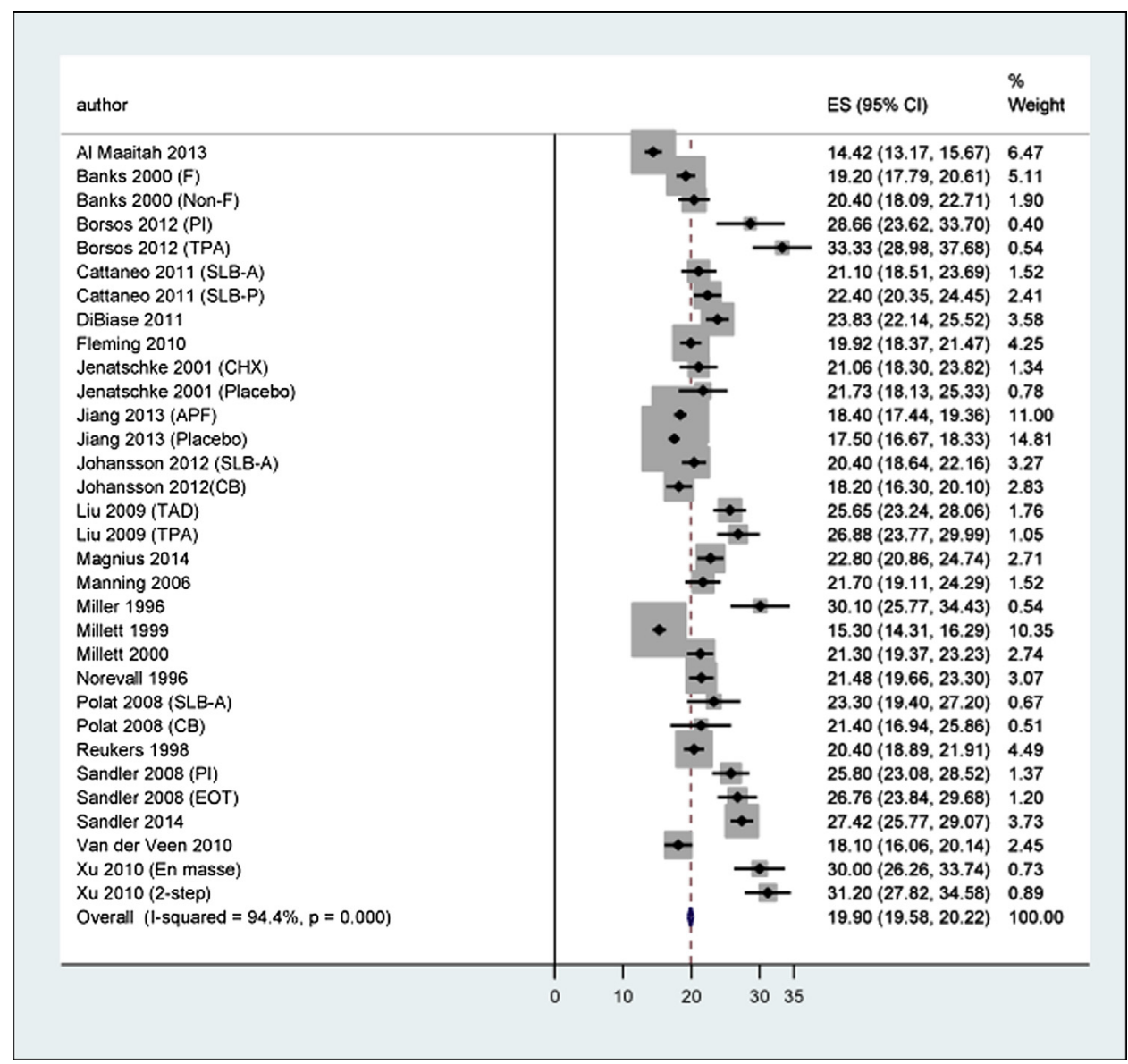

Fig 5. Forest plot for treatment duration excluding studies with low quality. ES, Effect size (mean difference).

\section{DISCUSSION}

On the basis of this systematic review, it appears that an average course of comprehensive orthodontic treatment with fixed appliances requires considerably less than 2 years (19.9 months). However, a wide range of treatment durations (14-33 months) were reported in the studies. This variation may relate to baseline and treatment-related differences among the studies, although important potential confounders were minimized by omitting studies involving adjunctive appliances, additional treatment phases, and combined orthodontic-surgical treatment. Clearly, the most significant arbiter of treatment duration appears to be the treating clinician, particularly his or her treatment planning decisions, standards, and finishing practices. This finding has previously been highlighted in retrospective studies investigating the potential impact of selfligating brackets, which reported marked differences in treatment duration, ranging from 15.8 to 31 months with conventional brackets. ${ }^{41,42}$
It is traditionally accepted that treatment with preadjusted edgewise appliances involves multiple phases, comprising initial alignment and leveling, overbite reduction, overjet reduction, space closure, and finishing and detailing of the occlusion. Therefore, average treatment durations of about 2 years appear reasonable. However, although these treatment phases may be considered as distinct entities, there is often considerable overlap between them, and mechanics including the use of fixed Class 11 correctors and fixed anterior biteplanes are becoming more accepted mechanisms of streamlining treatment without resorting to compromised objectives. ${ }^{43,44}$ Nevertheless, prospective research on the implications of the latter approaches in terms of overall treatment duration is lacking.

The present drive toward reducing orthodontic treatment duration may reflect an increasing awareness of cosmetic dental procedures and a growing uptake of orthodontic treatment by adults. ${ }^{45}$ Although social judgments may be less influential for children receiving 


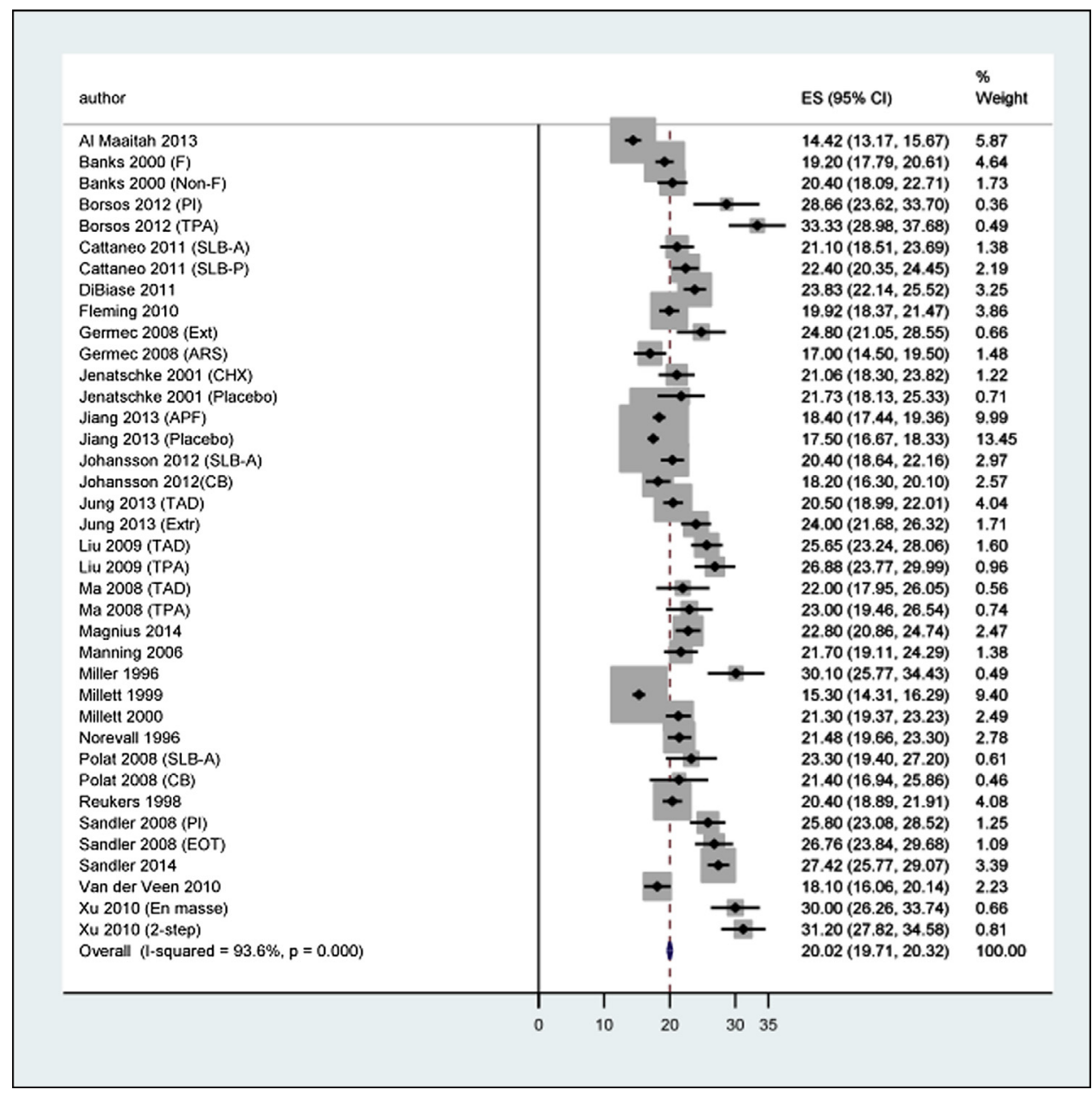

Fig 6. Forest plot for treatment duration including high- and low-quality studies. ES, Effect size (mean difference).

treatment, their impact on adults is more significant. ${ }^{46}$ This issue is compounded by an acceptance that biologic processes underpinning tooth movement are innately slower in adults. This set of circumstances has spawned the concept of short-term orthodontics, an approach that is undertaken either as a stand-alone procedure or to facilitate further minimally invasive cosmetic dentistry. Commonly, short-term orthodontics involves a truncated orthodontic treatment focusing on alignment of the anterior teeth, with a trade-off between shorter treatment and less complete occlusal correction. ${ }^{5}$ On the contrary, traditional orthodontic approaches seek to obtain ideal esthetic and functional results and maximize long-term stability. While a perfect occlusal outcome is often elusive in orthodontics, ${ }^{47}$ its achievement is contingent on adequate expression of tip and torque, combined with careful treatment planning and mechanics. ${ }^{48}$ Realizing these objectives is necessarily slow, with complete torque expression particularly time-consuming. Conversely, with short-term orthodontic treatment, a compromised result is often premeditated. Therefore, patients must be aware of the objectives and limitations of each option before embarking on treatment. ${ }^{8,49}$ If the expected treatment duration is a barrier to undertaking comprehensive treatment, on the basis of this review, it appears reasonable to suggest that comprehensive correction should not normally take much longer than 20 months.

Treatment duration can be influenced by a variety of factors. In particular, the severity of the malocclusion, extraction-based treatment, multidisciplinary treatment involving hypodontia or orthognathic surgery, and alignment of impacted teeth allied to operator experience and patient compliance may all be influential. We had hoped to consider the effects of extractions and age in this review, but because we included studies largely involving both a combination of extraction and nonextraction patients with broad age 
ranges, and because individual patient data were not available, this was not possible. It does, however, seem reasonable to assume that these variable would have some effect and for patients to be advised accordingly.

While the pooled estimate of treatment duration is most likely representative, it remains unclear as to what an acceptable threshold for treatment duration may be for either children or adults. It is likely that this figure fluctuates among patients. However, assuming that most adolescent patients are willing to undergo treatment for the average expected period of 20 months, it certainly appears that undertaking either compromised treatment or a blanket prescription of as yet unproven adjunctive nonsurgical or surgical procedures cannot at this stage be advocated. ${ }^{4}$ There may, however, be specific indications for these approaches and greater potential applications among adult patients, although it would appear important that patients should be apprised of the relative indications for adjunctive procedures and the limited evidence to underpin them. ${ }^{10,11}$

All included studies were undertaken in either a hospital or a university setting. Many of these patients may have been treated by trainees under supervision and had more complex malocclusions. ${ }^{51}$ it is therefore possible that the mean values obtained may constitute a slight overestimate of the overall duration of comprehensive orthodontic treatment. We had initially planned to assess the potential impact of treatment setting on the duration of treatment; however, because practice settings were not represented, this proved to be impossible.

As this review was carried out to gather epidemiologic data, rather than to undertake a comparative effectiveness review, a decision was made to include prospective studies in isolation. Consequently, potentially biased data from retrospective studies, whereby patients might have been selected on the basis of achieving a better, more efficient outcome, were omitted. ${ }^{52} \mathrm{~A}$ further methodologic complication was related to the fact that treatment duration was derived from some clinical trials in which the primary outcome was unrelated, with, for example, bracket failure or development of demineralization being common main outcomes. Consequently, bias inherent in these primary studies may not necessarily have directly affected estimates of treatment duration. The Cochrane risk of bias tool, however, was used to gauge bias associated with these studies to estimate inherent bias and study quality. Moreover, the Newcastle-Ottawa scale was used in the nonrandomized studies; a lack of uniformity in terms of the selection of quality assessment tools in systematic reviews is widely acknowledged. ${ }^{53}$

Overall, this meta-analysis involved many primary studies compared with similar reviews, with a median of 4 studies per meta-analysis recently shown in orthodontics. ${ }^{54}$ This may be because treatment duration seems to be commonly used as an important outcome measure in orthodontic trials. There is little consensus regarding the most influential orthodontic outcomes with an undue emphasis on clinician-centered and cephalometric outcomes. ${ }^{55}$ Clearly, however, treatment time does appear to be a key consideration for orthodontists and patients, particularly adults.

\section{CONCLUSIONS}

On the basis of this review, it is reasonable to assume that the average duration of comprehensive orthodontic treatment is less than 2 years. If alternative approaches to reduce the treatment time are undertaken, it would be sensible that these interventions or alternatives are chosen with an awareness of this yardstick.

\section{ACKNOWLEDGMENTS}

We thank the authors of the studies included in this systematic review for providing further information and data on their research.

\section{SUPPLEMENTARY DATA}

Supplementary data related to this article can be found at http://dx.doi.org/10.1016/j.ajodo.2015.09.020.

\section{REFERENCES}

1. Reitan K. Clinical and histologic observations on tooth movement during and after orthodontic treatment. Am J Orthod 1967;53: 721-45.

2. Krishnan V, Davidovitch Z. On a path to unfolding the biological mechanisms of orthodontic tooth movement. J Dent Res 2009; 88:597-608.

3. Mavreas D, Athanasiou AE. Factors affecting the duration of orthodontic treatment: a systematic review. Eur J Orthod 2008;30: 386-95.

4. Long H, Pyakurel U, Wang Y, Liao L, Zhou Y, Lai W. Interventions for accelerating orthodontic tooth movement: a systematic review. Angle Orthod 2013;83:164-71.

5. Maini A. Short-term cosmetic orthodontics for general dental practitioners. Br Dent J 2013;214:83-4.

6. Segal GR, Schiffman PH, Tuncay OC. Meta analysis of the treatment-related factors of external apical root resorption. Orthod Craniofac Res 2004;7:71-8.

7. Johal A, Alyaqoobi 1, Patel R, Cox S. The impact of orthodontic treatment on quality of life and self-esteem in adult patients. Eur J Orthod 2015;37:233-7.

8. Chate RA. Truth or consequences: the potential implications of short-term cosmetic orthodontics for general dental practitioners. Br Dent J 2013;215:551-3.

9. Darendeliler MA, Zea A, Shen G, Zoellner H. Effects of pulsed electromagnetic field vibration on tooth movement induced by magnetic and mechanical forces: a preliminary study. Aust Dent $\mathrm{J}$ 2007;52:282-7. 
10. Hoogeveen EJ, Jansma J, Ren Y. Surgically facilitated orthodontic treatment: a systematic review. Am J Orthod Dentofacial Orthop 2014;145(4 Suppl):S51-64.

11. Woodhouse NR, DiBiase AT, Johnson N, Slipper C, Grant J, Alsaleh M, et al. Supplemental vibrational force during orthodontic alignment: a randomized trial. J Dent Res 2015;94:682-9.

12. Fleming PS, Fedorowicz Z, Johal A, El-Angbawi A, Pandis N. Surgical adjunctive procedures for accelerating orthodontic treatment. Cochrane Database Syst Rev 2015;CD010572.

13. Uribe F, Padala S, Allareddy V, Nanda R. Patients', parents', and orthodontists' perceptions of the need for and costs of additional procedures to reduce treatment time. Am J Orthod Dentofacial Orthop 2014;145(4 Suppl):S65-73.

14. Higgins JP, Altman DG, Gotzsche PC, Juni P, Moher D, Oxman AD, et al. The Cochrane Collaboration's tool for assessing risk of bias in randomised trials. BMJ 2011;343:d5928.

15. Wells GA, Shea B, O'Connell D, Peterson JE, Welch V, Losos M, et al. The Newcastle-Ottawa scale (NOS) for assessing the quality of nonrandomised studies in meta-analyses; 2000. Available at: http://www.ohri.ca/programs/clinical_epidemiology/oxford.asp. Accessed March 2, 2015.

16. Al Maaitah EF, Abu Omar AA, Al-Khateeb SN. Effect of fixed orthodontic appliances bonded with different etching techniques on tooth color: a prospective clinical study. Am J Orthod Dentofacial Orthop 2013;144:43-9.

17. Banks PA, Chadwick SM, Asher-McDade C, Wright JL. Fluoridereleasing elastomerics: a prospective controlled clinical trial. Eur J Orthod 2000;22:401-7.

18. Borsos G, Voko Z, Gredes T, Kunert-Keil C, Vegh A. Tooth movement using palatal implant supported anchorage compared to conventional dental anchorage. Ann Anat 2012;194:556-60.

19. Cattaneo PM, Treccani M, Carlsson K, Thorgeirsson T, Myrda A, Cevidanes $\mathrm{LH}$, et al. Transversal maxillary dento-alveolar changes in patients treated with active and passive self-ligating brackets: a randomized clinical trial using CBCT-scans and digital models. Orthod Craniofac Res 2011;14:222-33.

20. DiBiase AT, Nasr IH, Scott P, Cobourne MT. Duration of treatment and occlusal outcome using Damon3 self-ligated and conventional orthodontic bracket systems in extraction patients: a prospective randomized clinical trial. Am J Orthod Dentofacial Orthop 2011;139:e111-6.

21. Fleming PS, DiBiase AT, Lee RT. Randomized clinical trial of orthodontic treatment efficiency with self-ligating and conventional fixed orthodontic appliances. Am J Orthod Dentofacial Orthop 2010;137:738-42.

22. Germec D, Taner TU. Effects of extraction and nonextraction therapy with air-rotor stripping on facial esthetics in postadolescent borderline patients. Am J Orthod Dentofacial Orthop 2008;133: 539-49.

23. Jenatschke F, Elsenberger E, Welte HD, Schlagenhauf U. Influence of repeated chlorhexidine varnish applications on mutans streptococci counts and caries increment in patients treated with fixed orthodontic appliances. J Orofac Orthop 2001;62:36-45.

24. Jiang H, Hua F, Yao L, Tai B, Du M. Effect of 1.23\% acidulated phosphate fluoride foam on white spot lesions in orthodontic patients: a randomized trial. Pediatr Dent 2013;35:275-8.

25. Johansson K, Lundstrom F. Orthodontic treatment efficiency with self-ligating and conventional edgewise twin brackets: a prospective randomized clinical trial. Angle Orthod 2012;82:929-34.

26. Jung YR, Kim SC, Kang KH, Cho JH, Lee EH, Chang NY, et al. Placement angle effects on the success rate of orthodontic microimplants and other factors with cone-beam computed tomography. Am J Orthod Dentofacial Orthop 2013;143:173-81.
27. Liu YH, Ding WH, Liu J, Li Q. Comparison of the differences in cephalometric parameters after active orthodontic treatment applying mini-screw implants or transpalatal arches in adult patients with bialveolar dental protrusion. J Oral Rehabil 2009;36: 687-95.

28. Ma J, Wang L, Zhang W, Chen W, Zhao C, Smales RJ. Comparative evaluation of micro-implant and headgear anchorage used with a pre-adjusted appliance system. Eur J Orthod 2008;30:283-7.

29. Magnius M, Bazargani F. Effects of oil-based and oil-free enamel prophylactic agents on bracket failure-a prospective randomized clinical trial. Swed Dent J 2014;38:87-91.

30. Manning N, Chadwick SM, Plunkett D, Macfarlane TV. A randomized clinical trial comparing 'one-step' and 'two-step' orthodontic bonding systems. J Orthod 2006;33:276-83:discussion 256-7.

31. Miller JR, Mancl L, Arbuckle G, Baldwin J, Phillips RW. A three-year clinical trial using a glass ionomer cement for the bonding of orthodontic brackets. Angle Orthod 1996;66:309-12.

32. Millett DT, Nunn JH, Welbury RR, Gordon PH. Decalcification in relation to brackets bonded with glass ionomer cement or a resin adhesive. Angle Orthod 1999;69:65-70.

33. Millett DT, McCluskey LA, McAuley F, Creanor SL, Newell J, Love J. A comparative clinical trial of a compomer and a resin adhesive for orthodontic bonding. Angle Orthod 2000;70:233-40.

34. Norevall Ll, Marcusson A, Persson M. A clinical evaluation of a glass ionomer cement as an orthodontic bonding adhesive compared with an acrylic resin. Eur J Orthod 1996;18:373-84.

35. Polat O, Gokcelik A, Arman A, Arhun N. A comparison of white spot lesion formation between a self-ligating bracket and a conventional preadjusted straight wire bracket. World J Orthod 2008;9: e46-50.

36. Reukers EA, Sanderink GC, Kuijpers-Jagtman AM, van’t Hof MA. Radiographic evaluation of apical root resorption with 2 different types of edgewise appliances. Results of a randomized clinical trial. J Orofac Orthop 1998;59:100-9.

37. Sandler J, Benson PE, Doyle P, Majumder A, O'Dwyer J, Speight P, et al. Palatal implants are a good alternative to headgear: a randomized trial. Am J Orthod Dentofacial Orthop 2008;133:51-7.

38. Sandler J, Murray A, Thiruvenkatachari B, Gutierrez R, Speight P, O'Brien K. Effectiveness of 3 methods of anchorage reinforcement for maximum anchorage in adolescents: A 3-arm multicenter randomized clinical trial. Am J Orthod Dentofacial Orthop 2014;146: 10-20.

39. van der Veen $\mathrm{MH}$, Attin R, Schwestka-Polly R, Wiechmann D. Caries outcomes after orthodontic treatment with fixed appliances: do lingual brackets make a difference? Eur J Oral Sci 2010;118:298-303.

40. Xu TM, Zhang X, Oh HS, Boyd RL, Korn EL, Baumrind S. Randomized clinical trial comparing control of maxillary anchorage with 2 retraction techniques. Am J Orthod Dentofacial Orthop 2010;138: 544.e1-9:discussion 544-5.

41. Eberting JJ, Straja SR, Tuncay OC. Treatment time, outcome, and patient satisfaction comparisons of Damon and conventional brackets. Clin Orthod Res 2001;4:228-34.

42. Hamilton R, Goonewardene MS, Murray K. Comparison of active self-ligating brackets and conventional pre-adjusted brackets. Aust Orthod J 2008;24:102-9.

43. Deregibus A, Debernardi CL, Persin L, Tugarin V, Markova M. Effectiveness of a fixed anterior bite plane in Class 11 deep-bite patients. Int J Orthod Milwaukee 2014;25:15-20.

44. Zymperdikas VF, Koretsi V, Papageorgiou SN, Papadopoulos MA. Treatment effects of fixed functional appliances in patients with Class 11 malocclusion: a systematic review and meta-analysis. Eur J Orthod 2015;418-34. 
45. Scott $P$, Fleming $P$, DiBiase A. An update in adult orthodontics. Dent Update 2007;34:427-8,431-4, 436 passim.

46. Jeremiah HG, Bister D, Newton JT. Social perceptions of adults wearing orthodontic appliances: a cross-sectional study. Eur J Orthod 2011;33:476-82.

47. Kattner PF, Schneider BJ. Comparison of Roth appliance and standard edgewise appliance treatment results. Am J Orthod Dentofacial Orthop 1993;103:24-32.

48. Andrews LF. The six keys to normal occlusion. Am J Orthod 1972; 62:296-309.

49. Slater R, Hunt N. Providing a choice. Br Dent J 2013;214:325-6.

50. O'Brien K, Wright J, Conboy F, Appelbe P, Bearn D, Caldwell S, et al. Prospective, multi-center study of the effectiveness of orthodontic/orthognathic surgery care in the United Kingdom. Am J Orthod Dentofacial Orthop 2009;135:709-14.

51. Campbell CL, Roberts WE, Hartsfield JK Jr, Qi R. Treatment outcomes in a graduate orthodontic clinic for cases defined by the
American Board of Orthodontics malocclusion categories. Am J Orthod Dentofacial Orthop 2007;132:822-9.

52. Pandis N, Tu YK, Fleming PS, Polychronopoulou A. Randomized and nonrandomized studies: complementary or competing? Am J Orthod Dentofacial Orthop 2014;146: 633-40.

53. Seehra J, Pandis N, Koletsi D, Fleming PS. Use of quality assessment tools in systematic reviews was varied and inconsistent. J Clin Epidemiol 2015 [Epub ahead of print].

54. Koletsi D, Fleming PS, Eliades T, Pandis N. The evidence from systematic reviews and meta-analyses published in orthodontic literature. Where do we stand? Eur J Orthod 2015;37: 603-9.

55. Tsichlaki A, O'Brien K. Do orthodontic research outcomes reflect patient values? A systematic review of randomized controlled trials involving children. Am J Orthod Dentofacial Orthop 2014;146: 279-85. 